\title{
Pediatric Multiple Sclerosis-A Challenging Demyelinating Disease: Case Report and Brief Review of the Literature
}

\author{
Regina Célia Ajeje Pires de Albuquerque, ${ }^{1}$ Raquel Siqueira Leonel de Paula, ${ }^{2}$ \\ Manuelina Mariana Capellari Macruz Brito, ${ }^{3}$ \\ José Roberto Lopes Ferraz Filho, ${ }^{4}$ and Lucas Crociati Meguins ${ }^{5}$ \\ ${ }^{1}$ Division of Neuropediatrics and Metabolic Diseases, Department of Pediatrics and Pediatric Surgery, Hospital de Base da Faculdade \\ de Medicina de São José do Rio Preto (FAMERP), 5544 São José do Rio Preto, SP, Brazil \\ ${ }^{2}$ Department of Pediatrics and Pediatric Surgery, Hospital de Base da Faculdade de Medicina de São José do Rio Preto (FAMERP), \\ 5544 São José do Rio Preto, SP, Brazil \\ ${ }^{3}$ Department of Neurological Sciences, Hospital de Base da Faculdade de Medicina de São José do Rio Preto (FAMERP), \\ 5544 São José do Rio Preto, SP, Brazil \\ ${ }^{4}$ Department of Radiology, Hospital de Base da Faculdade de Medicina de São José do Rio Preto (FAMERP), \\ 5544 São José do Rio Preto, SP, Brazil \\ ${ }^{5}$ Department of Neurological Sciences, Hospital de Base da Faculdade de Medicina de São José do Rio Preto (FAMERP), \\ Rua Pedro Palotta, 101/31B, 15092-205 São José do Rio Preto, SP, Brazil
}

Correspondence should be addressed to Lucas Crociati Meguins, lucascrociati@libero.it

Received 16 April 2012; Accepted 21 June 2012

Academic Editors: A. Gedalia and M. Moschovi

Copyright ( 2012 Regina Célia Ajeje Pires de Albuquerque et al. This is an open access article distributed under the Creative Commons Attribution License, which permits unrestricted use, distribution, and reproduction in any medium, provided the original work is properly cited.

\begin{abstract}
Multiple sclerosis (MS) is an inflammatory, demyelinating, neurodegenerative disorder of the central nervous system (CNS) of unknown etiology. The peak onset is between age 20 and 40 years and usually affects more women than men. Although much knowledge has been achieved on the diagnosis and treatment of adult patients with MS, it remains a matter of debate and controversy in childhood. We present a case of MS in 9-year-old girl, review the current state of the knowledge on pediatric MS, and discuss the available tools for the diagnosis and treatment.
\end{abstract}

\section{Introduction}

Multiple sclerosis (MS) is described as an inflammatory, demyelinating, neurodegenerative disorder of the central nervous system (CNS) and is uncommonly seen in pediatric patients [1-4]. Pediatric MS represents about 2.2\% to $4.4 \%$ of all MS cases, in late childhood, affects more girls than boys, and is characterized by a relapsing-remitting course in almost cases [5-8]. Although, much knowledge has been achieved on the diagnosis and treatment of adult patients with MS, it remains a matter of debate and controversy in childhood.

The aim of the present report is to describe the case of 9-year-old girls with MS and discuss the current knowledge for the diagnosis of MS and the therapeutic possibilities.

\section{Case Report}

A 9-year-old girl was referred for neurological examination due to sudden onset of diplopia. The patient affirmed that after waking up on that morning, she started to feel visual impairment in which an object was seen as two while having both eyes open. She also said that the distance between the two objects enlarged when looking to the left. Her past medical and familial history was unremarkable for any degenerative or neurological disorder. General clinical assessment of the patient was found within normal limits. Neurological examination revealed convergent strabismus and no movement of the left lateral rectus muscle. The remainder of the exam was essentially normal, including 


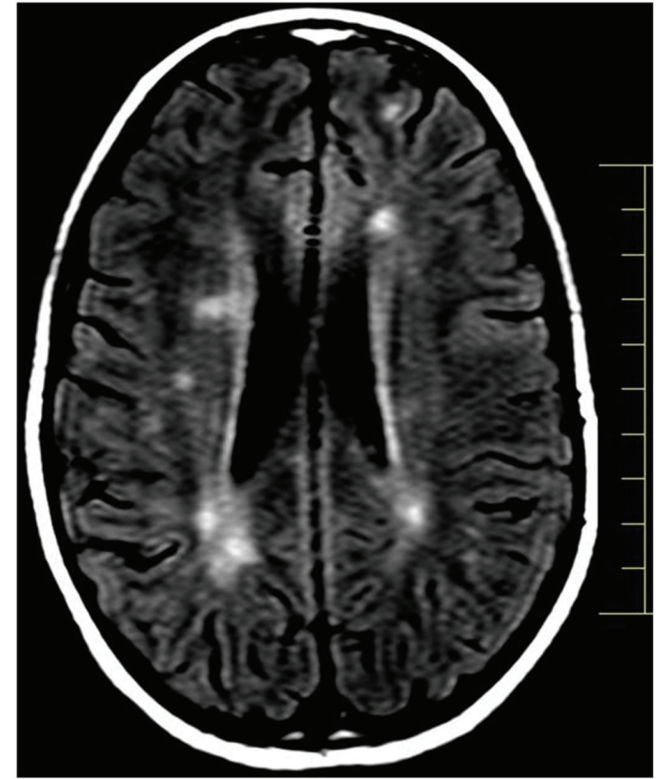

FIgURE 1: Brain MRI showing multiple periventricular lesions.

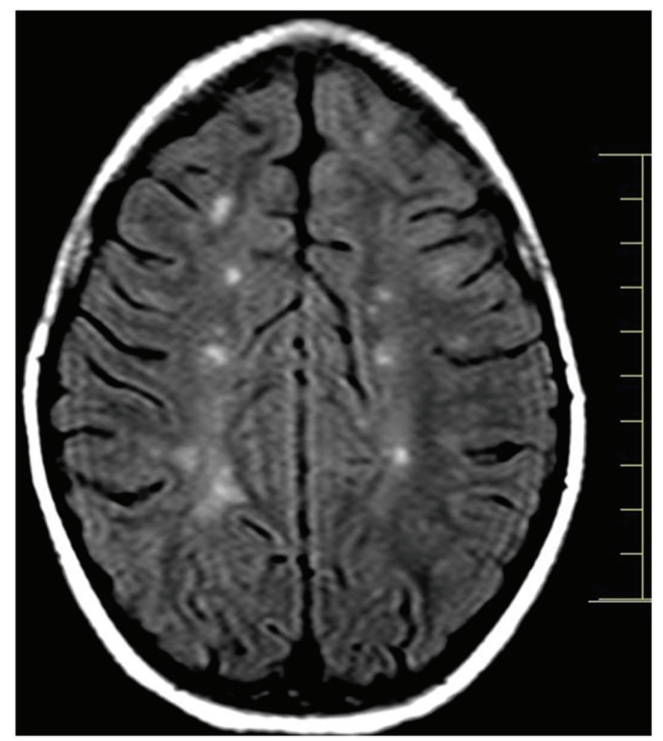

FIGURE 2: Brain MRI showing multiple juxtacortical lesions.

visual fields and routine ophthalmoscopy. Blood laboratory exams and electrocardiogram were found to be normal. Magnetic resonance imaging (MRI) of the brain showed multiple lesions affecting the periventricular, juxtacortical and infratentorial regions (Figures 1, 2, and 3). Brain MRI also showed gadolinium-enhancing and nonenhancing lesions (Figure 4). A cerebrospinal fluid analysis was unremarkable. As no other structural abnormalities were identified, a diagnosis of multiple sclerosis (MS) was made and the neurological symptom was attributed to it. Based on this diagnosis, we started pulse corticosteroid therapy with methylprednisolone during three days. The girl did not

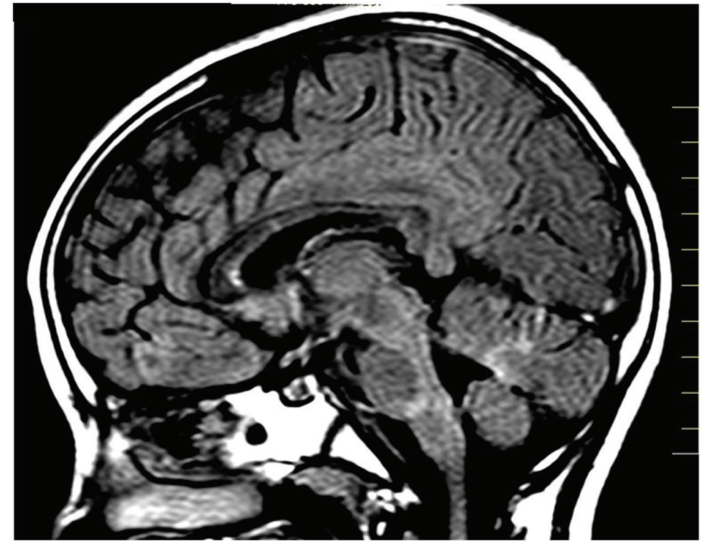

FIGURE 3: Brain MRI showing multiple infratentorial lesions.

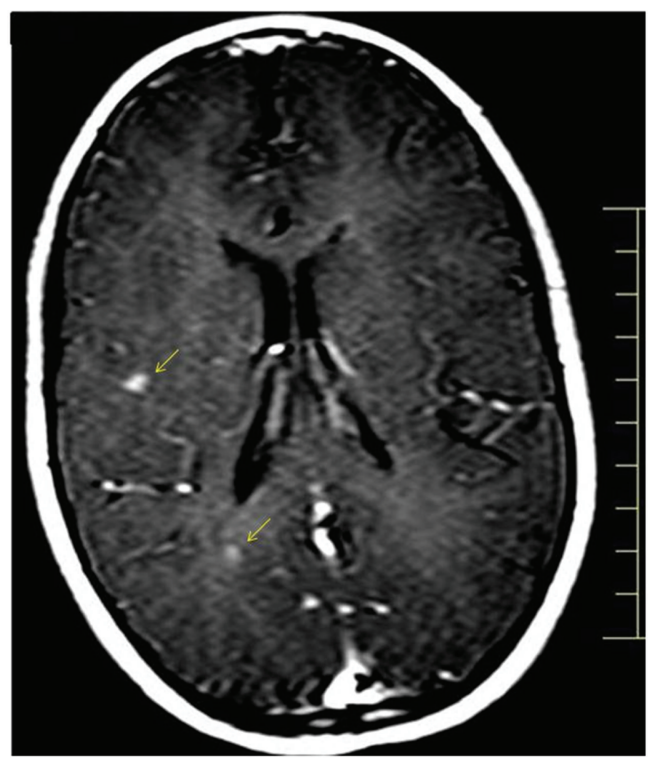

FIGURE 4: Brain MRI showing multiple gadolinium-enhancing lesions (arrows).

recover after 6 months of the diplopia and is currently being followed on outpatient appointments.

\section{Discussion}

Multiple sclerosis (MS) is characterized as an inflammatory autoimmune disorder of the central nervous system (CNS) in which the fatty myelin sheaths around the axons of the brain and spinal cord are damaged, leading to demyelination and scarring [9-11]. It was first described by Jean-Martin Charcot, a French researcher, in 1868 [12], and affects more women than men [13]. Its incidence if found to be $5.68 / 100.000$ per year and the pediatric population accounts for about $2.2 \%$ to $4.4 \%$ of all MS cases $[5-8,14]$. Pediatric MS represents a challenging diagnosis to those that deal with pediatric care, mainly because it presents proper and different characteristics from adults. 
Swanton and colleagues, in 2007 [15], and Montalban and colleagues, in 2010 [16], have made important contribution on the diagnosis of MS proposing simpler criteria allowing easer MRI evidence for dissemination in space (DIS) and dissemination in time (DIT) to be used in patients who present with clinically isolated syndrome (CIS), respectively. In May 2010, in Dublin, Ireland, on the third review of the McDonald criteria, the International Panel on the Diagnosis of Multiple Sclerosis (the Panel) not only accepted the simplified methods but also affirmed that the new criteria will also serve well for most pediatric MS patients, especially those with acute demyelination presenting as CIS [17].

According to the Panel [17], DIS can be demonstrated with at least 1 T2 lesion in at least 2 of 4 locations considered characteristic of MS: juxtacortical, periventricular, infratentorial, and spinal cord. Additionally, DIT can also be confirmed in patients with typical CIS with a single MRI study that demonstrates DIS and both asymptomatic gadolinium-enhancing and nonenhancing lesions. In the present report, our patient suddenly complained of diplopia resulting from left VI cranial nerve palsy consistent with a clinical isolated syndrome affecting the brainstem. Brain and spinal cord MRI study revealed multiple lesions consistent with DIS and DIT additional criteria to MS.

Currently available first-line diseases-modifying therapies to MS for adults, including interferon $\beta$ and glatiramer acetate, have not been approved by FDA for the treatment of children with MS $[1,18]$. However, it has been proved to be safe and well tolerated in pediatric population [19-21]. Probably, different biology in terms of drug metabolism, immune mechanisms, and incomplete maturity of the central nervous system is responsible for the diverse response to treatment in children; therefore further investigation is required to assess the better clinical approach of pediatric patients. Our patient did not present a good clinical response to pulse corticosteroid therapy. She is currently being followed on outpatient appointments.

In conclusion, given the distinct features and substantial variability of symptoms in pediatric patients, a high clinical awareness to the possibility of MS diagnosis is necessary, seeking help from experts in central nervous system demyelinating diseases.

\section{References}

[1] T. Chitnis, L. Krupp, A. Yeh et al., "Pediatric Multiple Sclerosis," Neurologic Clinics, vol. 29, no. 2, pp. 481-505, 2011.

[2] M. Absinta, M. A. Rocca, L. Moiola et al., "Cortical lesions in children with multiple sclerosis," Neurology, vol. 76, no. 10, pp. 910-913, 2011.

[3] M. Kamate, V. Chetal, V. Tonape, N. Mahantshetti, and V. Hattiholi, "Central nervous system inflammatory demyelinating disorders of childhood," Annals of Indian Academy of Neurology, vol. 13, no. 4, pp. 289-292, 2010.

[4] B. Banwell, A. Bar-Or, G. Giovannoni, R. C. Dale, and M. Tardieu, "Therapies for multiple sclerosis: considerations in the pediatric patient," Nature Reviews Neurology, vol. 7, no. 2, pp. 109-122, 2011.
[5] C. Renoux, S. Vukusic, Y. Mikaeloff et al., "Natural history of multiple sclerosis with childhood onset," The New England Journal of Medicine, vol. 356, no. 25, pp. 2603-2613, 2007.

[6] A. Ghezzi, V. Deplano, J. Faroni et al., "Multiple sclerosis in childhood: clinical features of 149 cases," Multiple Sclerosis, vol. 3, no. 1, pp. 43-46, 1997.

[7] B. Banwell, L. Krupp, J. Kennedy et al., "Clinical features and viral serologies in children with multiple sclerosis: a multinational observational study," The Lancet Neurology, vol. 6, no. 9, pp. 773-781, 2007.

[8] A. Ghezzi, "Therapeutic strategies in childhood multiple sclerosis," Therapeutic Advances in Neurological Disorders, vol. 3, no. 4, pp. 217-228, 2010.

[9] J. Río, M. Comabella, and X. Montalban, "Multiple sclerosis: current treatment algorithms," Current Opinion in Neurology, vol. 24, no. 3, pp. 230-237, 2011.

[10] J. H. Noseworthy, C. Lucchinetti, M. Rodriguez, and B. G. Weinshenker, "Multiple sclerosis," The New England Journal of Medicine, vol. 343, no. 13, pp. 938-952, 2000.

[11] D. M. Wingerchuk, C. F. Lucchinetti, and J. H. Noseworthy, "Multiple sclerosis: current pathophysiological concepts," Laboratory Investigation, vol. 81, no. 3, pp. 263-281, 2001.

[12] M. Clanet, “Jean-Martin Charcot. 1825 to 1893," International MS Journal/MS Forum, vol. 15, no. 2, pp. 59-61, 2008.

[13] C. Jobin, C. Larochelle, H. Parpal, P. K. Coyle, and P. Duquette, "Gender issues in multiple sclerosis: an update," Women's Health, vol. 6, no. 6, pp. 797-820, 2010.

[14] J. Benito-León, "Are the prevalence and incidence of multiple sclerosis changing?" Neuroepidemiology, vol. 36, no. 3, pp. 148-149, 2011.

[15] J. K. Swanton, A. Rovira, M. Tintore et al., "MRI criteria for multiple sclerosis in patients presenting with clinically isolated syndromes: a multicentre retrospective study," The Lancet Neurology, vol. 6, no. 8, pp. 677-686, 2007.

[16] X. Montalban, M. Tintoré, J. Swanton et al., "MRI criteria for MS in patients with clinically isolated syndromes," Neurology, vol. 74, no. 5, pp. 427-434, 2010.

[17] C. H. Polman, S. C. Reingold, B. Banwell et al., "Diagnostic criteria for multiple sclerosis: 2010 revisions to the McDonald criteria," Annals of Neurology, vol. 69, no. 2, pp. 292-302, 2011.

[18] E. A. Yeh, T. Chitnis, L. Krupp et al., "Pediatric multiple sclerosis," Nature Reviews Neurology, vol. 5, no. 11, pp. 621631, 2009.

[19] A. Ghezzi, M. P. Amato, M. Capobianco et al., "Treatment of early-onset multiple sclerosis with intramuscular interferon $\beta$ 1a: long-term results," Neurological Sciences, vol. 28, no. 3, pp. 127-132, 2007.

[20] B. Kornek, G. Bernert, C. Balassy, J. Geldner, D. Prayer, and M. Feucht, "Glatiramer acetate treatment in patients with childhood and juvenile onset multiple sclerosis," Neuropediatrics, vol. 34, no. 3, pp. 120-126, 2003.

[21] N. L. Kuntz, D. Chabas, B. Weinstock-Guttman et al., "Treatment of multiple sclerosis in children and adolescents," Expert Opinion on Pharmacotherapy, vol. 11, no. 4, pp. 505-520, 2010. 


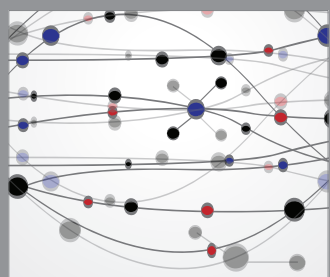

The Scientific World Journal
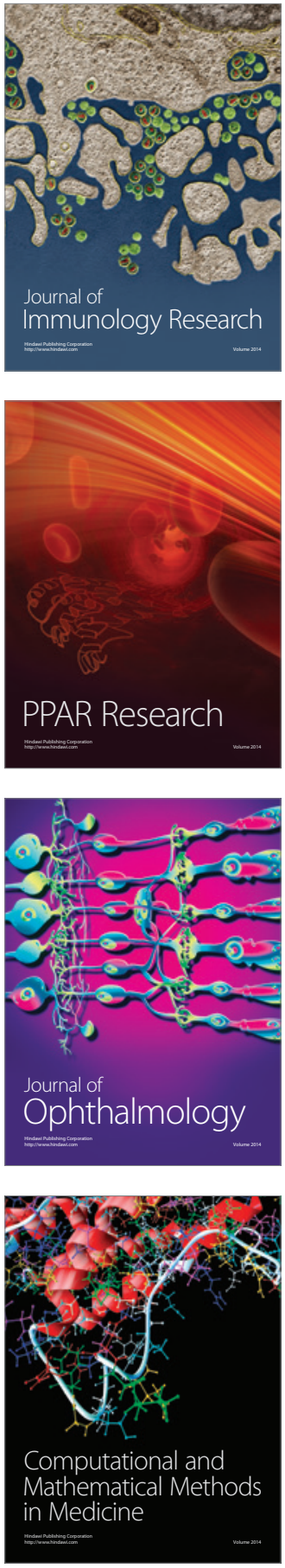

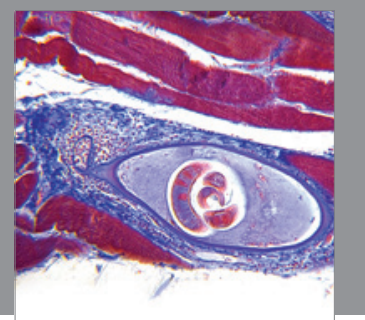

Gastroenterology

Research and Practice
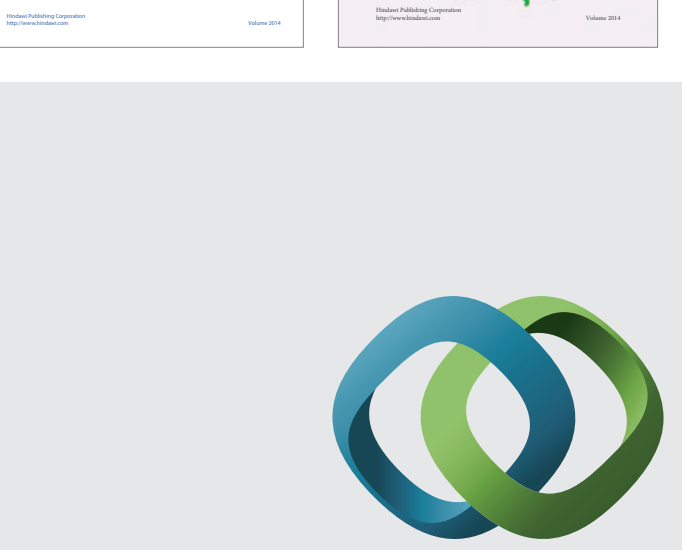

\section{Hindawi}

Submit your manuscripts at

http://www.hindawi.com
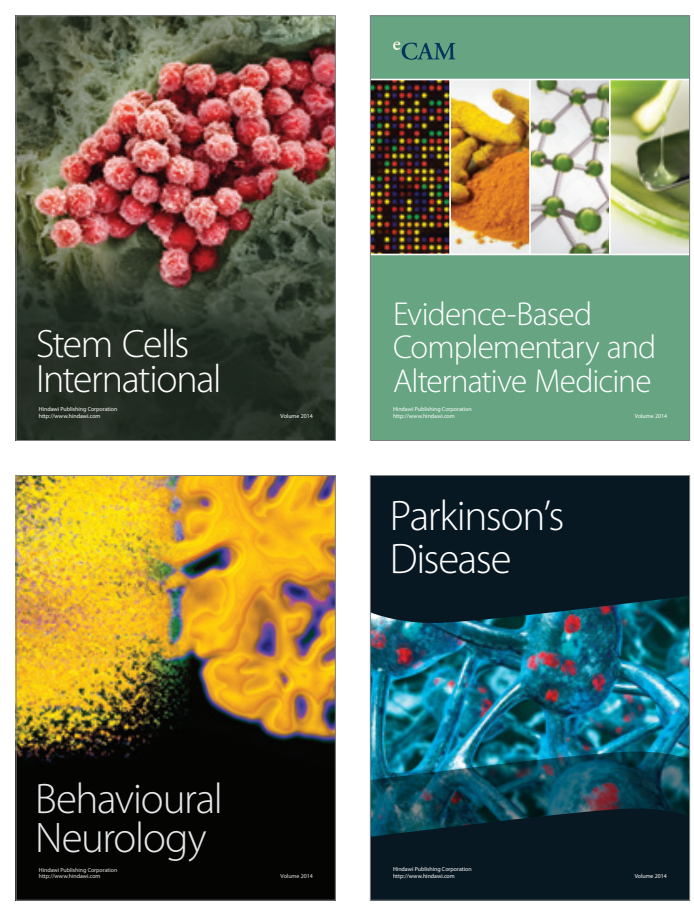

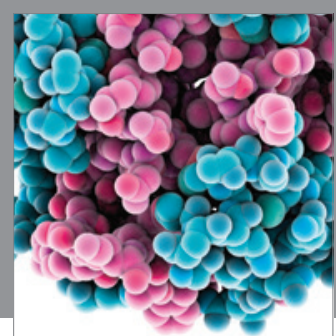

Journal of
Diabetes Research

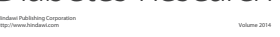

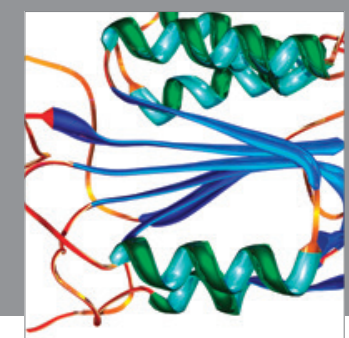

Disease Markers
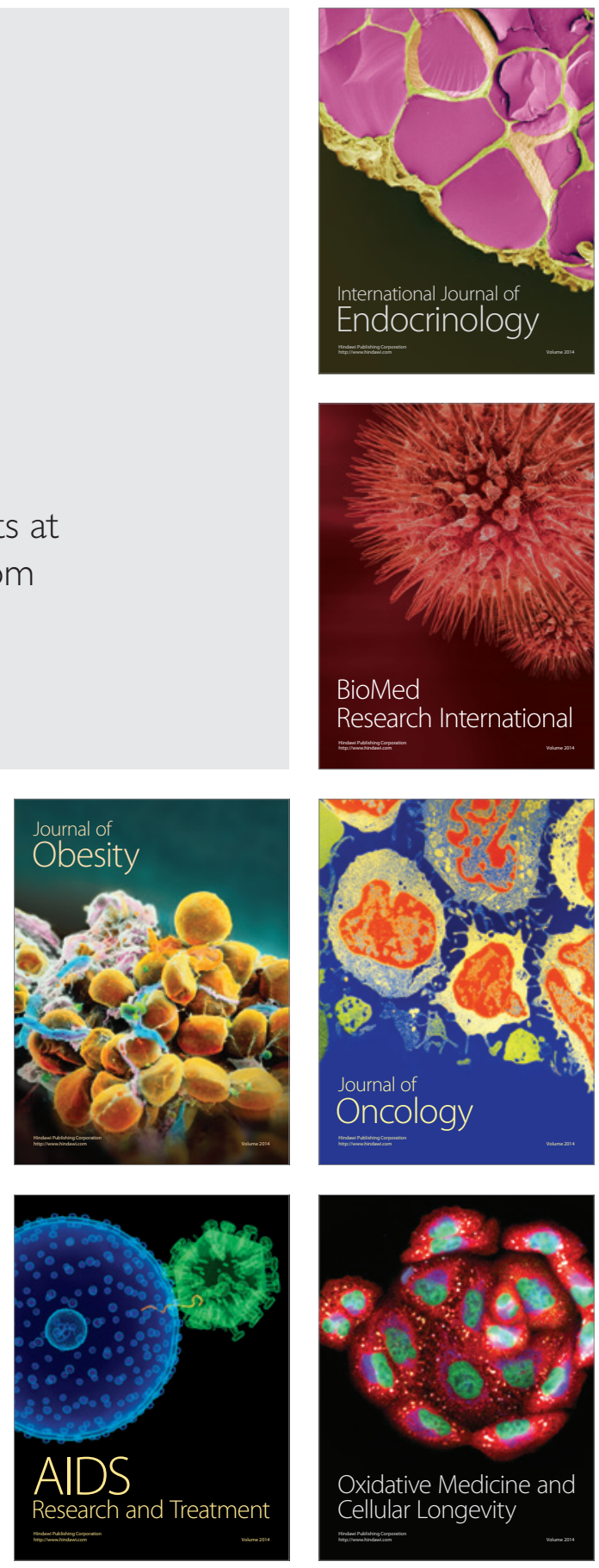